\title{
Dopamine Activation Preserves Visual Motion Perception Despite Noise Interference of Human V5/MT
}

\author{
Nada Yousif, ${ }^{1}$ Richard Z. Fu, ${ }^{2}$ Bilal Abou-El-Ela Bourquin, ${ }^{2}$ Vamsee Bhrugubanda, ${ }^{2}{ }^{\circledR S i m o n}$ R. Schultz, ${ }^{3}$ \\ and ${ }^{\circ}$ Barry M. Seemungal ${ }^{2}$ \\ ${ }^{1}$ Imperial College Neuromodulation Group and 2Neuro-Otology Group, Charing Cross Hospital Campus, Imperial College, London W6 8RF, \\ United Kingdom, and ${ }^{3}$ Centre for Neurotechnology and Department of Bioengineering, South Kensington Campus, Imperial College, London SW7 2AZ, \\ United Kingdom
}

When processing sensory signals, the brain must account for noise, both noise in the stimulus and that arising from within its own neuronal circuitry. Dopamine receptor activation is known to enhance both visual cortical signal-to-noise-ratio (SNR) and visual perceptual performance; however, it is unknown whether these two dopamine-mediated phenomena are linked. To assess this, we used single-pulse transcranial magnetic stimulation (TMS) applied to visual cortical area V5/MT to reduce the SNR focally and thus disrupt visual motion discrimination performance to visual targets located in the same retinotopic space. The hypothesis that dopamine receptor activation enhances perceptual performance by improving cortical SNR predicts that dopamine activation should antagonize TMS disruption of visual perception. We assessed this hypothesis via a double-blinded, placebo-controlled study with the dopamine receptor agonists cabergoline (a D2 agonist) and pergolide (a D1/D2 agonist) administered in separate sessions (separated by 2 weeks) in 12 healthy volunteers in a William's balance-order design. TMS degraded visual motion perception when the evoked phosphene and the visual stimulus overlapped in time and space in the placebo and cabergoline conditions, but not in the pergolide condition. This suggests that dopamine D1 or combined D1 and D2 receptor activation enhances cortical SNR to boost perceptual performance. That local visual cortical excitability was unchanged across drug conditions suggests the involvement of long-range intracortical interactions in this D1 effect. Because increased internal noise (and thus lower SNR) can impair visual perceptual learning, improving visual cortical SNR via D1/D2 agonist therapy may be useful in boosting rehabilitation programs involving visual perceptual training.

Key words: dopamine; dopamine agonist; MT/V5; rehabilitation; TMS; visual cortex; visual motion

Significance Statement

In this study, we address the issue of whether dopamine activation improves visual perception despite increasing sensory noise in the visual cortex. We show specifically that dopamine D1 (or combined D1/D2) receptor activation enhances the cortical signalto-noise-ratio to boost perceptual performance. Together with the previously reported effects of dopamine upon brain plasticity and learning (Wolf et al., 2003; Hansen and Manahan-Vaughan, 2014), our results suggest that combining rehabilitation with dopamine agonists could enhance both the saliency of the training signal and the long-term effects on brain plasticity to boost rehabilitation regimens for brain injury.

\section{Introduction}

Noise is a feature of the nervous system and originates both externally from the variability of the stimulus itself and internally from neuronal activity (Arieli et al., 1996; Cavanaugh et al., 2015)

Received Dec. 14, 2015; revised May 24, 2016; accepted June 27, 2016.

Author contributions: N.Y., S.R.S., and B.M.S. designed research;N.Y., R.Z.F., B.A.-E.-E.-B., V.B., S.R.S., and B.M.S. performed research; N.Y., S.R.S., and B.M.S. analyzed data; N.Y., R.Z.F., S.R.S., and B.M.S. wrote the paper.

N.Y. was supported by a medical research grant from the Bupa Health Foundation. B.M.S. was supported by an Academy of Medical Sciences and Health Foundation Fellowship and by the Medical Research Council UK. This research was also supported by the NIHR Biomedical Research Centre at Imperial College Healthcare NHS Trust and Imperial College London.

The authors declare no competing financial interests.

This article is freely available online through the J Neurosci Author Open Choice option.
The brain deals with noisy inputs by averaging information over repeated trials and by using an expectation about the structure of noise in sensory inputs (Ermentrout et al., 2008). Alterations in internal noise in cerebral cortical circuits may be important clinically because alterations in internal noise may play a critical role in mediating recovery in perceptual functioning after brain in-

Correspondence should be addressed to Barry M. Seemungal, Neuro-Otology Group, Charing Cross Hospital Campus, Imperial College, London W6 8RP, UK. E-mail: b.seemunga@@ic.ac.uk.

DOl:10.1523/JNEUROSCI.4452-15.2016

Copyright $\odot 2016$ Yousif et al.

This is an Open Access article distributed under the terms of the Creative Commons Attribution License Creative Commons Attribution 4.0 International, which permits unrestricted use, distribution and reproduction in any medium provided that the original work is properly attributed. 
jury (Cavanaugh et al., 2015). It follows that interventions that modulate internal noise in neural cerebral cortical circuits may aid recovery from brain injury and potentially boost rehabilitation of patients.

Dopaminergic activation improves the cortical neuronal signal-to-noise ratio (SNR) (Winterer and Weinberger, 2004; Kroener et al., 2009) and enhances visual perceptual performance (Müller et al., 1998; Noudoost and Moore, 2011). However, it is unknown whether this dopamine enhancement of visual perceptual performance results from a dopamine-induced increase in SNR. This predicts that dopaminergic activation would reduce the disruptive effects of increased neuronal noise on perceptual performance. Providing evidence linking dopaminergic enhancement of SNR and perceptual functioning could support a mechanistic-based approach to pharmacological boosting of rehabilitation programs.

We set out to assess the impact of dopaminergic modulation of perceptual functioning in healthy volunteers but reproducing high internal noise states (and lowered SNR) by applying focal single-pulse transcranial magnetic stimulation (TMS) to the visual cortex (Ruzzoli et al., 2010). When applied to the visual cortex at sufficient intensity, TMS degrades visual perceptual performance transiently for visual targets located in the retinotopic space of the stimulated brain region. The hypothesis that dopaminergic enhancement of visual perceptual performance results from a cortical SNR improvement would predict that dopamine activation should reverse (or limit) the expected noise-related degradation of visual perceptual performance artificially imposed by TMS (or, by extrapolation, by disease).

To test this prediction, we assessed the impact of TMS on a standard visual perceptual task requiring subjects to indicate the net direction of motion (right vs left) of a cloud of dots moving in coherent motion embedded within a cloud of randomly moving dots (Braddick, 1974; random dot kinematogram or RDK). The RDK task difficulty can be titrated by changing the proportion of coherent versus random motion dots (Fig. 1A). Applying TMS to visual cortex disrupts performance in the RDK task when at least two conditions are concurrently satisfied: (1) when the visual space occupied by the RDK overlaps with the retinotopic space of the stimulated brain region, which is indicated by the location of the TMS-evoked "phosphene" (a perceived flash of light occurring after visual cortical TMS stimulation) overlapping the location of the RDK; and (2) when there is adequate temporal overlap between RDK onset and the TMS pulse. When these two conditions are met, the same brain region is stimulated at the same time by both the visual stimulus (RDK) and the TMS pulse.

A further open question is whether any observed dopaminergic effect on visual cortical SNR is mediated locally (i.e., in the visual cortex). Recent single-neuron animal data suggest that the effects of dopamine upon visual cortical functioning, including its effect upon SNR, are mediated by mechanisms distant to the visual cortex (Zaldivar et al., 2014). For example, modulating dopamine D1 activity in primate prefrontal cortex affects visual cortical neuronal responsiveness (Noudoost and Moore, 2011). Therefore, in addition to using TMS to introduce noise to degrade visual performance, we also used it to assess any change in local (i.e., V5/MT) baseline visual cortical responsivity that might arise after dopaminergic activation and thus determine whether changes in visual perceptual performance relate to changes in local SNR (i.e., in visual cortex).

\section{Materials and Methods}

\section{General method}

To model the situation of increased neural noise in the visual cortex, we applied single-pulse TMS. Because TMS modulates cortical function transiently ( $\sim 50 \mathrm{~ms}$; Cowey and Walsh, 2001), nonspecific effects of TMS can be controlled by varying the overlap between a visual stimulus and the time of TMS pulse onset. Figure 1 demonstrates the experimental method by which we assessed the effect of TMS upon visual perceptual function across space and time.

We ran two Experiments: A and B. The results of Experiment A were used to refine the stimulus parameters for Experiment B. In Experiment $\mathrm{A}$, in two separate sessions, we compared the impact of threshold versus suprathreshold left V5/MT cortical TMS on visual motion discrimination. Experiment A showed that suprathreshold TMS was more disruptive of visual motion discrimination performance than threshold TMS.

Therefore, in Experiment B, we used suprathreshold TMS at V5/MT to disrupt visual motion discrimination performance and assessed how this TMS disruption was affected by pharmacological dopamine receptor activation. There were three drug conditions in Experiment B: (1) placebo (lactose, a disaccharide sugar), (2) cabergoline (a dopamine D2 receptor agonist) at $1 \mathrm{mg}$, and (3) pergolide (a dopamine D1 and D2 agonist) at $500 \mu \mathrm{g}$. The doses of cabergoline and pergolide chosen took into account the doses used in prior studies, the dose-related side effects of both drugs, and the need to maintain clinical dose equivalents between pergolide and cabergoline (Reichmann et al., 2003). Previous studies have used cabergoline doses of up to $1.5 \mathrm{mg}$ (Norbury et al., 2013), requiring a pergolide dose equivalent of $1000 \mu \mathrm{g}$, a dose likely to cause excessive symptoms. We thus chose an intermediate cabergoline dose of $1 \mathrm{mg}$, providing a clinical equivalent for pergolide of $500 \mu \mathrm{g}$. To avoid drug side effects, we adapted a clinical protocol (Jansen et al., 2001) used for the rapid titration of pergolide therapy in Parkinson's disease (PD). Therefore, all participants were prescribed domperidone $20 \mathrm{mg} 3$ times per day for $3 \mathrm{~d}$ before testing and were given additional domperidone doses on the day of testing (up to $60 \mathrm{mg}$ that day). To maintain double blinding, the drug capsules (containing placebo, pergolide, or cabergoline) were made up by a pharmacological laboratory and packaged in identical inert shells. Participants were tested on three separate sessions on different days with sessions separated by a minimum of $14 \mathrm{~d}$ to account for the drug half-lives in the systemic circulation (Fariello, 1998; Rascol, 1999; Del Dotto et al., 2003; Agúndez et al., 2013). To balance order effects, we randomized the order of drug administration using a William's balanced order design. The randomization list was drawn up by a college staff member not involved in the study and provided to the pharmacy, which then dispensed the drugs on a named-volunteer basis according to the list.

We minimized within-subject, intersession performance variability, and between-subject variability in three ways. First, the variability in TMS location was minimized using neuronavigation (see "TMS target localization" section below). Second, the variability in visual cortical responsiveness (e.g., due to learning effects or differences in vigilance) was controlled by calibrating the TMS intensity at every session (see "TMS-evoked phosphene threshold determination" section below), and, finally, the baseline visual motion coherence was titrated to a fixed visual perceptual response level at the start of every session (see "Baseline visual motion threshold determination" section below). Furthermore, we asked participants to complete a self-administered questionnaire on vigilance after drug administration, in which they were asked to indicate on a line where they felt themselves to be between two extreme states as follows: alert - drowsy; calm - excited; strong - feeble; muzzy - clearheaded; well-coordinated - clumsy; lazy - energetic; contented - discontented; troubled - tranquil; mentally slow - quick-witted; tense - relaxed; attentive - dreamy; incompetent - proficient; happy - sad; aggressive friendly; interested - bored; and withdrawn - outgoing.

This method has been used previously to measure participants' subjective feelings before and after sleep (Herbert et al., 1976). The scores were quantified by measuring the distance for each response and converting it into a percentage of the total length of the line. 
A
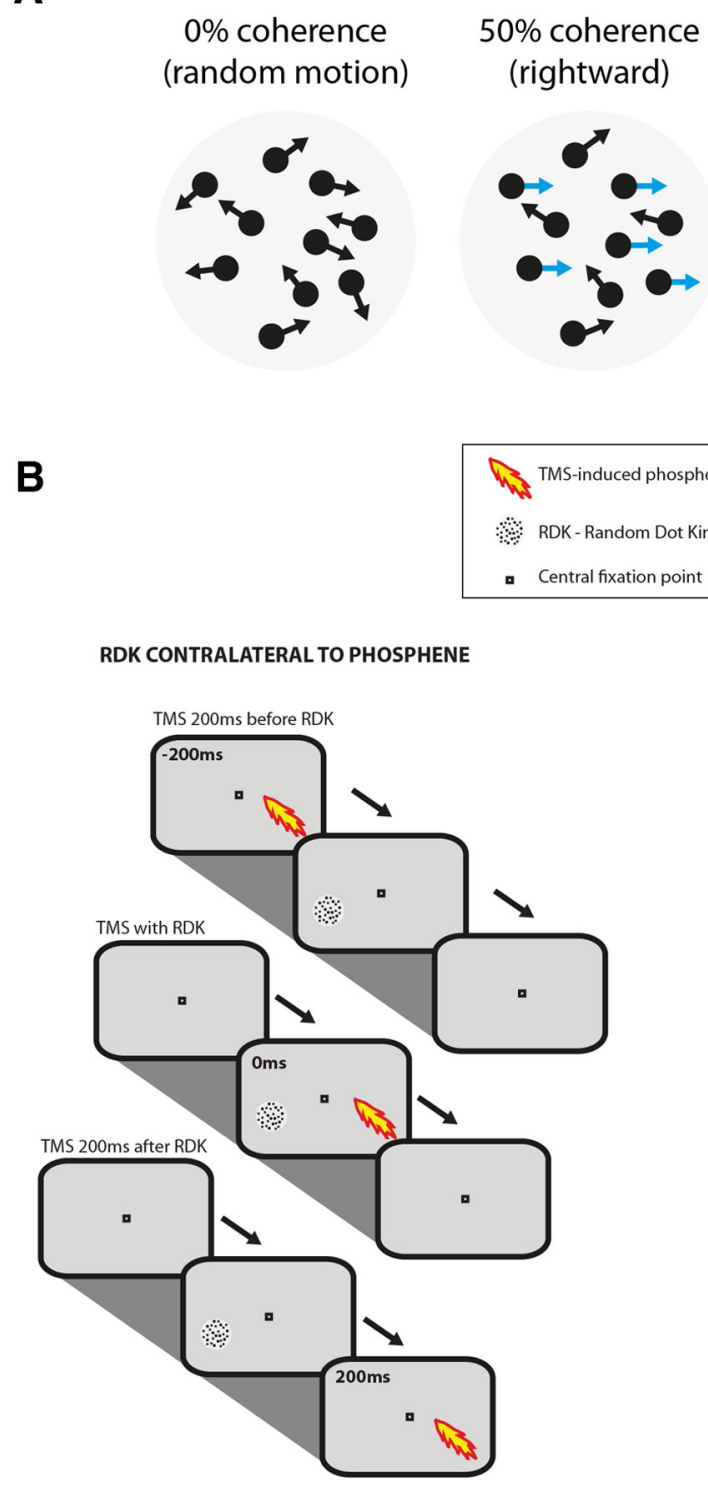
$100 \%$ coherence (rightward)

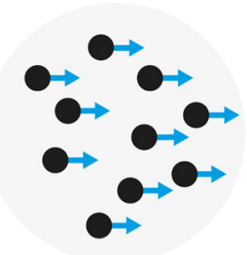

B

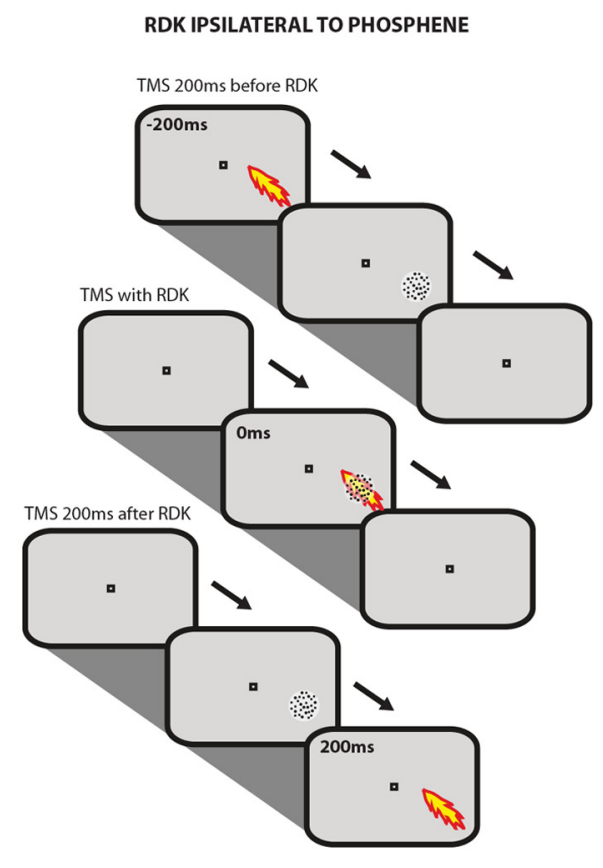

C

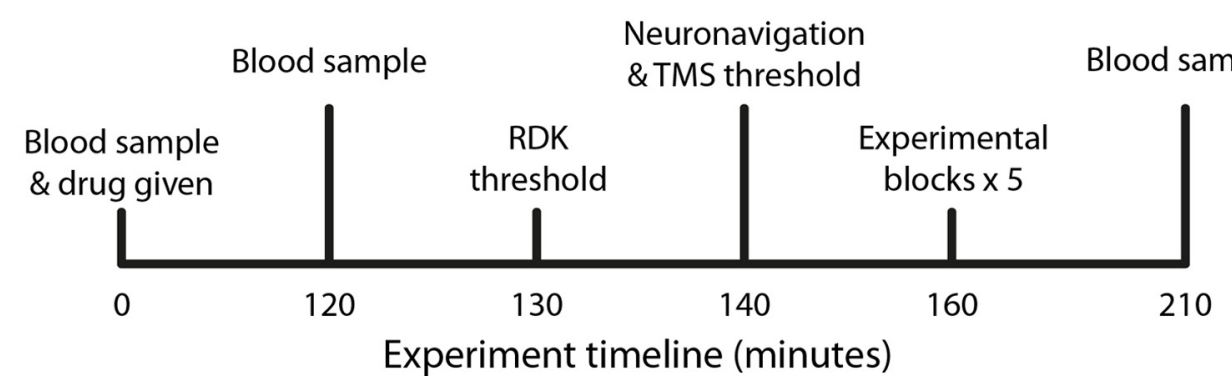

Figure 1. Methods. $A$, Schematic of the visual stimuli used throughout the experiments. Moving dots with a given coherence (percentage of dots moving either to the left or the right with the remainder moving with random directions) were presented in $5^{\circ} \times 5^{\circ}$ of visual angle aperture on a black screen for $200 \mathrm{~ms}$. The coherence of dot motion remained constant across trials and equal numbers of trials with leftward or rightward motion were presented. $\boldsymbol{B}$, Each trial consisted of a visual stimulus (at time 0 ) and a TMS pulse (lightening bolt) that was presented before, coincident with (Experiments 1 and 2), or after (Experiment 2 only) the onset of the visual stimulus. Dots appeared randomly on the nonoverlapping or overlapping visual space. C, Single session in Experiment 2.

TMS target localization

Single-pulse TMS was delivered using a MagPro X100 stimulator (MagVenture) via an MC-B70 Butterfly coil $(169 \times 112 \times 33 \mathrm{~mm})$ held in place over the region of interest by an adjustable coil holder. Because previous TMS studies had suggested a relative lateralization of motion processing within the left hemisphere (Stewart et al., 2001; Beckers and Homberg, 1992), this experiment used left V5/MT for all stimulation.

Area V5/MT was initially located functionally via the induction of moving phosphenes, a proven method used previously in studies of phosphene perception (Seemungal et al., 2013; Stewart et al., 2001). Briefly, a 
physical landmark over the left hemisphere $3 \mathrm{~cm}$ dorsal and $5 \mathrm{~cm}$ lateral to the inion was used as an initial starting point. With subjects in the dark and with their eyes open, the coil was moved manually around this region until subjects reliably perceived moving phosphenes in the right lower quadrant of their visual field, overlapping a region where subsequent visual stimuli would be presented in the experiment. Once area V5/MT was functionally located, its specific $3 \mathrm{D}$ coordinates relative to the subject's cerebral cortex were obtained with a neuronavigation system (BrainVoyager) using individual T1-weighted MRI brain scans. BrainVoyager QX 2.6.1 (Brain Innovation) was used to construct 3D head surface and left-hemisphere brain models ("meshes") from structural T1-weighted 3 tesla MRI scans of subjects' brains. This setup allowed the identified V5/MT stimulation point to be saved as a location on the head surface, so the maintenance of the same target could be ensured throughout the experiment and across sessions. This consistency across sessions of targeting the same stimulated area V5/MT in a given subject further reduced response variability.

\section{TMS-evoked phosphene threshold determination}

At the beginning of each experimental session, we calibrated the required TMS intensity. For Experiment A, we determined the TMS intensity that induced phosphenes with a probability of 50\% (threshold) and $79 \%$ (suprathreshold). For Experiment B, we only determined the suprathreshold intensity that elicited a $79 \%$ phosphene probability. Subjects were asked to fixate on a fixation cross on an otherwise dark screen. After every TMS pulse, they indicated "yes" or "no" as to whether they observed a phosphene via button press. An adaptive QUEST staircase procedure (Watson and Pelli, 1983) was used over 40 trials to converge on the threshold/suprathreshold TMS intensity. This calibration procedure was run twice, first as a practice block and then to yield the coherence values used for the experimental session. We also asked subjects to indicate where on the screen they observed the phosphene and this position was used for the presentation of the visual stimulus (see next section).

\section{Visual motion stimuli (RDK)}

The visual motion stimuli consisted of a black background with a $5^{\circ}$ diameter "cloud" of 50 circular moving dots with dots of angular diameter $2^{\circ}$ ( 4 pixels) and dot velocity of $12^{\circ} \%$ s. The dot cloud was presented for $200 \mathrm{~ms}$ to either the left or right of the midline in a randomized balanced order. The right hemifield visual stimulus location was programmed to overlie that of the subject's observed phosphene. The visual stimulus in the left hemifield was positioned at the same latitude and at equal distance from the midline relative to the right hemifield visual stimulus.

The visual stimulus consisted of an RDK with two populations of moving dots: one moving coherently to the right or left and another moving randomly. The relative ease of detecting the coherent population dot direction (right vs left) was modulated by altering the number of coherent moving dots to those moving randomly. The visual stimuli were presented on a 22 inch flat cathode ray tube display (Mitsubishi SuperBright Diamondtron DP2070SB) with a resolution of $1024 \times 768$ pixels and a refresh rate of $85 \mathrm{~Hz}$. PsychToolbox (Brainard, 1997; Pelli, 1997) was used to present the experimental stimuli, trigger the TMS, and record subject responses.

\section{Baseline visual motion threshold determination}

The proportion of coherently moving dots in the RDK was titrated so that subjects correctly identified the coherent dot motion direction with a $79 \%$ probability at baseline. This was done immediately after obtaining the TMS threshold determination. This procedure obtained the subject's threshold of perception for motion in each direction (right and left) before the experiment. The same adaptive QUEST staircase algorithm (Watson and Pelli, 1983) was used as was used for the TMS threshold determination. This calibration procedure involved 80 trials (40 in the left visual field and 40 in the right visual field) and was run twice, first as a practice and then to yield the coherence values used for the experimental session.

\section{General procedure}

Subjects sat on a padded, height-adjustable chair in a darkened laboratory. They were dark adapted for $30 \mathrm{~min}$ and the room luminance level was kept constant across all sessions. Subjects stabilized their head on a fixed chin rest, with their eyes $40 \mathrm{~cm}$ from the monitor screen. After aligning the TMS to the subject's left V5/MT using neuronavigation, the subject's TMS phosphene threshold was obtained. The coherence values for rightward and leftward global dot motion were then ascertained.

There were 32 trials per block in Experiment A and 48 trials per block in Experiment B. Each trial consisted of the presentation of the visual stimulus of moving dots (200 ms duration), with TMS applied at different times relative to the onset of the visual stimulus (see individual procedures below). Subjects were instructed to indicate with a button press via a two-alternate forced-choice design whether they perceived the dot motion direction as rightward or leftward. Subjects were required to fixate upon a continuously present, central fixation square of size $0.5^{\circ} \times$ $0.5^{\circ}$. In all trials, TMS was applied to the left cerebral hemisphere. In contrast, the visual stimulus was presented randomly to either the right or left visual field. In addition, the net coherent dot motion direction within the RDK was randomized between right and left. Dot motion direction and the three different times of TMS were randomized and balanced within a single block. The response was prompted by the fixation square changing into a question mark and subjects were instructed to respond as quickly as possible. Time between each trial was $5 \mathrm{~s}$, with a single block lasting $\sim 6.5 \mathrm{~min}$. Breaks were taken as necessary between blocks, but subjects were required to remain seated and were kept in the dark.

\section{Experiment $A$}

Subjects. Twelve subjects ( 9 male) mean age 25 years \pm 4 years with normal or corrected-to-normal vision comprising students and staff of Imperial College London participated in the study after informed consent. Approval for the study was granted by the Charing Cross Hospital Research Ethics Committee.

Procedure for Experiment A. Subjects completed one session of eight experimental blocks of 32 trials each, totaling 256 trials for the whole experiment. A session was initiated by determining the TMS threshold at $50 \%$ (threshold condition) and then at 79\% (suprathreshold condition) and a visual threshold at $79 \%$. The visual thresholds were determined twice to allow the subject one block as practice. Each experimental block was run with either threshold or suprathreshold TMS, the order of the blocks was alternated, and this in turn was counterbalanced across subjects. A single trial consisted of the presentation of the visual stimulus of moving dots (200 ms duration), with TMS asynchronously applied at either $200 \mathrm{~ms}$ before or simultaneous with the onset of the visual stimulus. The TMS time, dot direction, and visual presentation side was randomized and balanced across conditions.

\section{Experiment $B$}

Subjects. Twelve healthy male volunteers (mean age: $25 \pm 4$ years) with normal or corrected-to-normal vision comprising students and staff of Imperial College London participated in the study. One of these subjects had participated in Experiment A, with experiments separated by 11 months.

Prolactin assay. Dopamine inhibits the secretion of prolactin by the pituitary gland into the systemic circulation (Ben-Jonathan, 1985). Changes in serum prolactin level will thus reflect the systemic bioavailability of the ingested dopamine agonist for which absorption can be variable. Subjects provided a baseline blood sample for serum prolactin, after which they were administered one of the three randomized drugs depending on their preassigned drug sequence. Dopamine agonists can cause side effects such as somnolence and nausea (Frucht et al., 1999) by stimulating dopamine receptors within the chemoreceptor trigger zone of the area postrema, which, crucially, is outside of the blood-brain barrier (Miller and Leslie, 1994; Borison, 1986; Stefanini and ClementCormier, 1981). The peripherally acting dopamine D2 antagonist domperidone, which does not cross the blood-brain barrier, suppresses dopamine-related nausea without affecting central dopaminergic activity (Laduron and Leysen, 1979). It is standard clinical practice to pretreat patients with domperidone before administering dopamine agonists to reduce nausea (Jansen et al., 2001). In our study, all subjects took oral domperidone ( $20 \mathrm{mg} 3$ times/d for $3 \mathrm{~d}$ ) before testing. Note that one of 
the dopamine agonists, cabergoline, is a specific dopamine D2 agonist, so domperidone, a selective D2 antagonist, will antagonize the cabergoline effect in the periphery (but not in the brain). Because the pituitary gland is perfused with systemic blood, the prolactin-suppressing effect of cabergoline is attenuated by domperidone. In contrast, pergolide is a D1 and D2 agonist, so it still has a potent prolactin suppressing effect via its D1 action despite the presence of the D2 blockade by domperidone.

Procedure for Experiment B. After baseline blood testing and drug administration, the volunteers waited in a quiet room for $2 \mathrm{~h}$ to allow absorption of the drug. During this period, they could read or work on a computer. Serum prolactin was again sampled $2 \mathrm{~h}$ after administration because the peak plasma concentration of pergolide is reached after 1-3 $\mathrm{h}$ and that for cabergoline after 2-3 h (Agúndez et al., 2013). A final prolactin sample was obtained at the end of the testing session, typically at $3.5 \mathrm{~h}$ after drug administration.

After neuronavigation setup, determination of the TMS intensity level and titration of the visual motion coherence level, the subjects completed five blocks of 48 trials each, totaling 240 trials per session, and, as detailed in "General method," each subject participated in three sessions, one per drug condition. A single trial consisted of the presentation of the visual stimulus of moving dots (200 ms duration), with TMS applied asynchronously at $200 \mathrm{~ms}$ before, synchronous with, or $200 \mathrm{~ms}$ after the onset of the visual stimulus. As before, the side of the RDK presentation (left or right of the midline), the direction of coherent dot motion (leftward or rightward) within the RDK cloud, and the onset times of TMS were randomized and balanced across all 48 trials in a single block. All pretest calibration and the testing itself lasted $\sim 90 \mathrm{~min}$.

\section{Data analysis}

Task performance in identifying the direction of coherent motion within the RDK was quantified as the percentage correct. To correct for nonspecific effects of TMS, a response gain was calculated as the percentage correct for the condition in which the RDK and phosphene were overlapping (i.e., both RDK and phosphene in right hemifield) divided by the percentage correct obtained when the RDK and phosphene were nonoverlapping (i.e., RDK in left hemifield and phosphene in right hemifield). The data were analyzed using MATLAB (The MathWorks) and statistical calculations were performed using SPSS software with a significance level set at $p<0.05$. Two-tailed $t$ test results are reported as $t(n-1)=$ the test statistic, the $p$-value. Appropriate ANOVAs were used for within-subject analyses and multivariate analyses were undertaken when between-subject and group comparisons were being made.

\section{Drift diffusion model}

It was hypothesized that any dopamine-related enhancement of perceptual performance is related to an improvement in the visual cortical SNR. Therefore, the subjects' decision making process was modeled on the two-alternative forced choice task (of choosing the net RDK coherent motion direction) as a random walk process using a drift-diffusion model (Ratcliff and McKoon, 1988), which incorporates a measure of signal noise. At each time step during the decision making period, the model posits an accumulation of evidence that is integrated until a choice is made when a boundary is reached. The evidence accumulation is noisy and therefore the process is stochastic. The model can be described by the following equation:

$$
\chi_{n+1}=\chi_{n}+\delta+\varepsilon
$$

where $x_{t}$ represents the accumulated evidence at time $t, x_{t-1}$ at time $t-1$, $\delta$ is the drift rate representing the speed at which the boundaries are approached, and $\varepsilon$ represents Gaussian noise with variance $\sigma$. In the model, a correct response is made when the accumulated evidence reaches or surpasses a threshold level and an incorrect response is made when the opposite boundary is reached.

The model was fitted to each participant's performance (percentage correct and reaction time) for the placebo and pergolide conditions at simultaneous TMS and visual stimulus presentation only and for both overlapping (RDK and phosphene on the same hemifield) and nonoverlapping (RDK and phosphene on opposite hemifields) conditions. Three versions of the model were run: (1) in the three-parameter model, we allowed the noise $\square$ No spatial overlap of phosphene \& RDK Spatial overlap of phosphene \& RDK

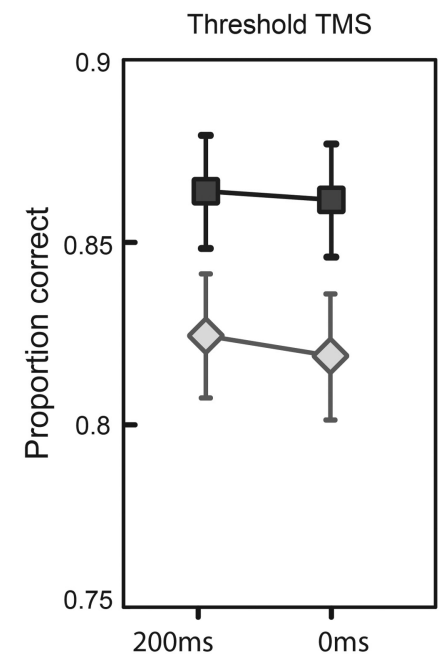
Suprathreshold TMS

(TMS before) (simultaneous)

Time of TMS relative to visual stimulus onset

Figure 2. Results of Experiment 1.A, Overall effect of TMS on response rate in Experiment 1. In the case when the TMS acted on the same visual space as the visual stimulus (overlapping), the correct response rate was suppressed compared with when the TMS and the visual stimulus did not overlap. This was consistent for threshold (left panel) and suprathreshold TMS (right panel) but most prominent for the Oms condition with suprathreshold TMS (right panel).

parameter, the drift speed, and the boundary parameters to all vary; (2) in the two-parameter model, only the noise and the drift speed parameters were allowed to vary; and (3) in the one-parameter model, we allowed only the drift speed parameter to vary. The fminsearch algorithm in MATLAB was used to minimize the sum of the $\chi^{2}$ for the percentage correct response and the reaction time. Finally, to assess which model best fit the data, the $\chi^{2}$ measure for each of the three models, normalized by the number of the fit parameters on a tri-plot, were fitted (Cavanaugh et al., 2002; El-Shamayleh and Movshon, 2011; see Fig. 6). The distance from the external edge shows which model best fits the data as displayed in Figure 6 via isometric and side projections.

\section{Results}

\section{Experiment A}

Consistent with the literature (Amassian et al., 1989), we found that supratheshold TMS (79\% probability of a phosphene report) impaired visual discrimination performance $\left(t_{(11)}=2.436, p=\right.$ 0.033 ) when the visual stimulus and the TMS-evoked phosphene occurred in the same visual space at the same time. Figure 2 shows the percentage correct response rate for spatially overlapping and nonoverlapping stimuli. It can be seen that, at both intensities, TMS impairs performance for visual stimuli that overlap phosphenes in place and time compared with visual stimuli that do not overlap phosphene location (and/or time of onset). A $2 \times 2 \times$ 2 repeated-measures ANOVA (factors: laterality, right vs left; TMS strength, threshold vs suprathreshold; and time, TMS 200 $\mathrm{ms}$ before and simultaneous with visual stimulus) of the percentage correct data showed a significant effect of laterality $(F=$ $6.031, p=0.032$ ) and a significant interaction of laterality versus time versus TMS strength $(F=3.796, p=0.038)$.

In summary, TMS to area V5/MT impairs visual motion discrimination performance when the visual stimulus overlaps the TMS-evoked phosphene in space and time and this effect is more prominent for suprathreshold TMS. 

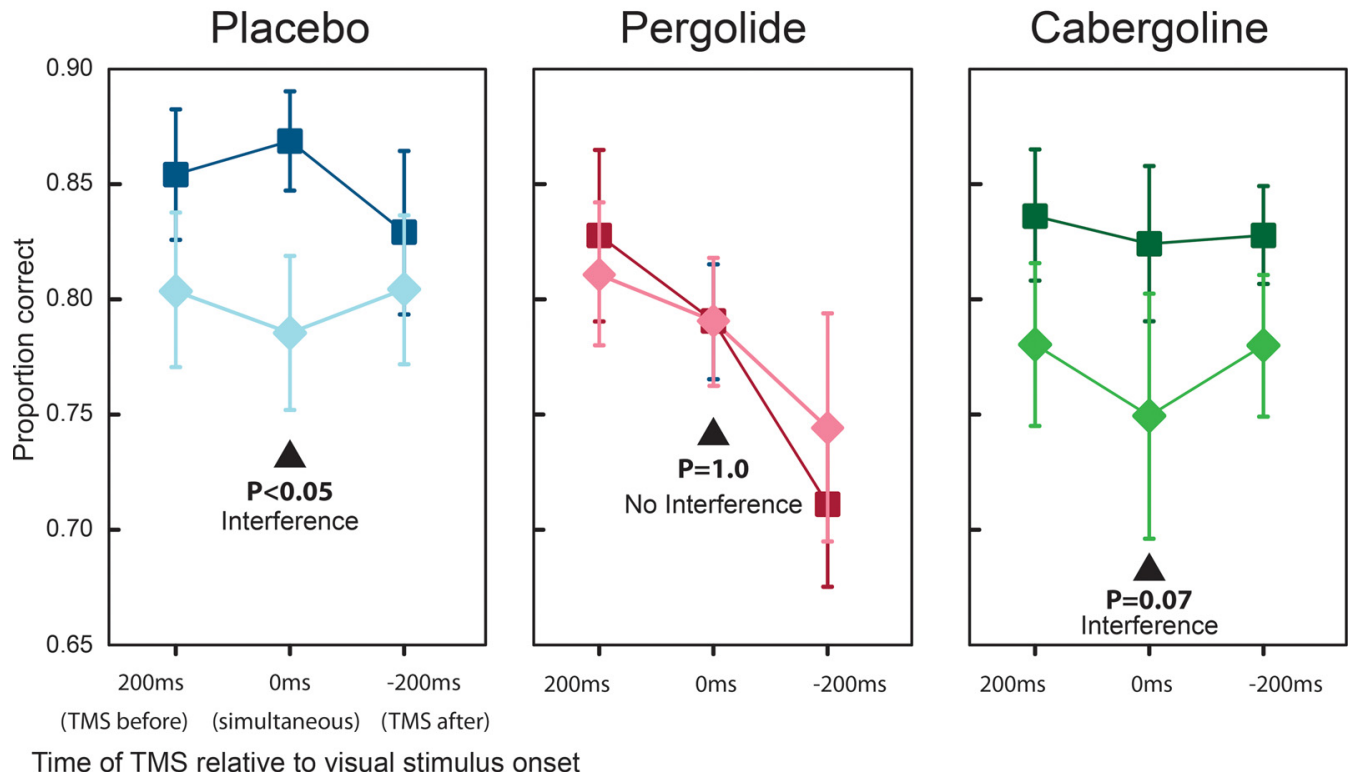

Time of TMS relative to visual stimulus onset

Figure 3. Results of Experiment 2. Shown is the effect of TMS on response rate under the different drug conditions in Experiment 2 . In the placebo case, we see the same effect as in Experiment 1, with TMS lowering the response rate to overlapping stimuli compared with nonoverlapping stimuli. This effect is also seen under the cabergoline condition. However, when subjects took pergolide, we find two effects: an overall suppression of response rate plus an improvement of the response to overlapping stimuli.

\section{Experiment B}

In Experiment B, we used the same experimental setup as Experiment A with three notable differences. First, we used a single TMS intensity set at suprathreshold intensity (79\% probability of a phosphene report). Second, we used three TMS onset latencies, $200 \mathrm{~ms}$ before, simultaneous with, and $200 \mathrm{~ms}$ after visual stimulus onset. Finally, all subjects performed the task three times in different sessions under different pharmacological conditions (i.e., pergolide, cabergoline, and placebo).

For each of the drug conditions (Fig. 3), we performed a repeated-measures $2 \times 3$ ANOVA with factors laterality of the RDK position relative to the visual hemifield (i.e., right or left) and time of TMS onset relative to the RDK onset (i.e., TMS 200 $\mathrm{ms}$ before, after, and simultaneously with the RDK).

For the placebo condition, we found that TMS impaired RDK motion discrimination performance (Fig. 3) when the TMSinduced phosphene and the RDK were simultaneously in the same visual space (the right hemifield, i.e., the overlapping condition). Specifically, we found a significant main effect of laterality $\left(F_{(1,11)}=\right.$ 6.01; $p=0.032$ ), but not time, and there was no interaction. We performed a single post hoc paired $t$ test at $t=0 \mathrm{~ms}$ (given the a priori expectation on this time point), finding a significant difference at $p<0.05$. Therefore, in the placebo condition in Experiment B, our data supported previous findings in the literature that TMS disrupts visual motion perception when the TMS and the visual motion stimulus coincide in space and time.

For the cabergoline condition, we found a similar disrupting effect of TMS at $t=0 \mathrm{~ms}$ with a significant main effect of laterality $\left(F_{(1,11)}=6.48 ; p=0.027\right)$ but not time. A single post hoc paired test at $t=0 \mathrm{~ms}$ showed a nonsignificant $(p=0.07)$ disruption of visual motion perception by TMS for RDK stimuli that were coincident with the TMS impulse in time and space.
In stark contrast, in the pergolide condition, we found no main effect of laterality ( $p=0.81$ ). Although not indicated given the nonsignificance of the ANOVA, for the purposes of comparison, we found that a 2-tailed $t$ test between the percentage correct for the right versus left hemifield RDKs at $t=0 \mathrm{~ms}$ gave a $p=1.0$. Furthermore, a power calculation gave a sample size of $>15,000$ per sample to enable the chance of finding a significant difference in performance for RDKs in the different hemifields at $t=0 \mathrm{~ms}$. In summary, in the pergolide condition, we found no evidence for any difference between the RDK performance between the two hemifields despite the disrupting effect of TMS on the right hemifield performance at $t=0 \mathrm{~ms}$. Note that we found a significant main effect of time $\left(F_{(2,10)}=5.00 ; p=0.017\right)$. This effect was likely related to a generalized alerting effect of TMS, which produces a loud click. A TMS click occurring before the visual stimulus (200 ms condition) will thus increase readiness for the visual stimulus compared with the $0 \mathrm{~ms}$ or $-200 \mathrm{~ms}$ condition, in which the TMS click occurs after the visual stimulus.

We also noted, however, that the overall performance in the pergolide condition was reduced compared with the placebo and the cabergoline conditions for both overlapping and nonoverlapping stimuli. This general reduction in performance during the pergolide condition was related to the reduced vigilance (sleepiness is a recognized side effect of pergolide administration); the group vigilance scores (where $0 \%$ represents maximum vigilance and $100 \%$ minimum vigilance) were $47 \%$ for pergolide, $27 \%$ for cabergoline, and $25 \%$ for placebo, with that for pergolide being different from that for placebo $\left(t_{(10)}=-2.591, p=0.027\right)$. Despite this, Figure 4 shows that there were no differences in baseline phosphene detection or visual perception between the three drug conditions (one-way ANOVA for each task: TMS: $F=$ 0.009, $p=0.991$; left visual stimuli: $F=0.07, p=0.993$; right 


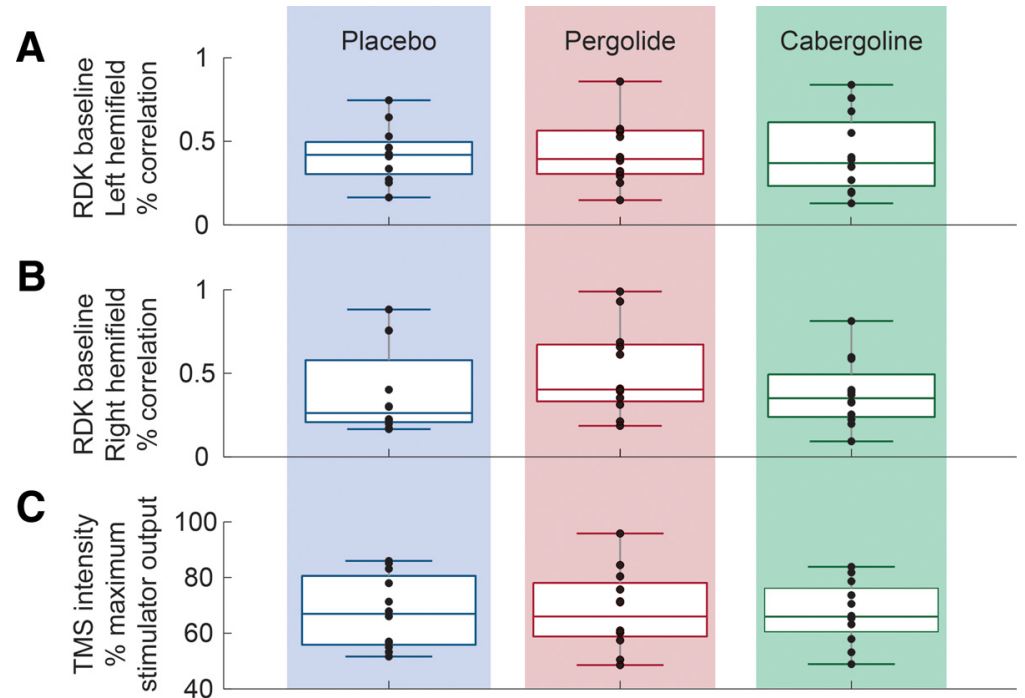

Figure 4. Baseline RDK visual motion coherence (percentage) for RDKs presented in the left visual space $(\boldsymbol{A})$ or right visual space $(\boldsymbol{B})$ and baseline TMS intensity $(\boldsymbol{C})$ for all three drug conditions (with TMS intensity expressed as a percentage of stimulator maximum). At each of the 36 experimental sessions ( 12 subjects $\times 3$ different drug sessions), baseline TMS intensity and RDK coherence was titrated to predetermined values (see Materials and Methods) to reduce intersubject and intersession variability. Baseline TMS intensity and RDK coherence were not significantly different across the three drug conditions, including for RDKs presented in the left visual space $(\boldsymbol{A})$ or right visual space $(\boldsymbol{B})$. Therefore, any difference across drug conditions cannot be due to differences in baseline visual cortex V5/MT excitability (which is correlated with the TMS intensity required to elicit phosphenes at a fixed probability) or RDK coherence.

visual stimuli: $F=1.132, p=0.335)$. That the baseline assessments were obtained after drug administration for each session separately shows that drug administration did not affect either baseline visual cortical excitability or visual motion detection performance without TMS.

The bioavailability of pergolide was assessed by measuring the fall in serum prolactin concentration obtained at three time points within a session: on arrival, $2 \mathrm{~h}$ after drug administration, and on completion of the experiment. We found that only pergolide caused a significant change in prolactin (Fig. 5A) and this was a $78 \%$ drop $(p=0.0042)$. Note that, for the reasons explained in the Materials and Methods section, the coadministration of domperidone blocked the prolactin-lowering effect of cabergoline (but had no effect on cabergoline's central action), so, as expected, we found no effect on prolactin levels with cabergoline administration.

If pergolide's effect on visual perceptual performance is mediated by its dopamine agonist activity, then this predicts that the gain in performance at time $t=0 \mathrm{~ms}$ (i.e., percentage correct for right $\mathrm{RDK} /$ left $\mathrm{RDK}$ ) achieved by subjects in the pergolide condition should scale with the relative fall in serum prolactin. Figure $5 B$ shows the gain of the response in the pergolide condition (overlapping/nonoverlapping percentage correct) for the $0 \mathrm{~ms}$ condition versus the change in the prolactin level (end of experiment measure). The plot shows that the visual perceptual response gain was correlated with the fall in serum prolactin (one-tailed $t$ test: $r^{2}=0.29, t_{(10)}=2.021, p=0.035$ ). This indicates that, the greater the pergolide-related dopamine activation, the better the participants responded to visual stimuli that overlapped in time and space with the effects of TMS. Note that one subject was omitted from this analysis because his baseline prolactin level in the pergolide condition was more $>4$ SDs away from the group mean (note that the baseline measures were taken before any pergolide administration). This particular result was confirmed to be spurious because this subject's baseline serum prolactin levels were within the normal range of the other subjects for the other two conditions (placebo and cabergoline).

\section{Drift diffusion model}

We fitted a drift diffusion model (see Materials and Methods) to our data from Experiment $\mathrm{B}$ to assess which model parameters (drift speed, noise level, and/or boundary distance) were affected by dopamine receptor activation. The results are plotted in terms of the corrected $\chi^{2}$ values for each subject's data fit to each model on a tri-plot (Cavanaugh et al., 2002; El-Shamayleh and Movshon, 2011). This plot shows the three $\chi^{2}$ values as the end position of a vector in $3 \mathrm{D}$ space such that the best fit is given by the edge closest to the resulting position. For our results, all of the data points are closest to the three-parameter model edge (Fig. 6), indicating that this model provides the best account of the data given the differing number of parameters between the models. Furthermore, on moving from the three- to the two-parameter model (i.e., removing the boundary parameter), there is comparatively less impact on fit compared with moving from the two- to the one-parameter model (i.e., removing noise). This indicates that the modulation of noise variance by dopaminergic activation plays an important role in the observed pergolide effect upon visual motion perceptual performance.

\section{Discussion}

Recent evidence supports the notion that dopamine improves the SNR in visual cortical networks (Winterer and Weinberger, 2004) and enhances perceptual performance (Noudoost and Moore, 2011); however, it was not clear whether these dopamine-related effects were linked through a common mechanism. We found that dopaminergic receptor activation via pergolide (a D1/D2 agonist), but not cabergoline (a D2 agonist), maintained visual perceptual performance despite a TMS-induced increased SNR; that pergolide's effect on visual perceptual performance was dose dependent; and that, in a drift-diffusion model, the noise parameter was critical for explaining pergolide's effect upon perceptual performance. Together, our experimental and modeled data strongly link pergolide administration with improved perceptual performance via a dopamine-related enhancement of neuronal SNR.

Although we found a specific effect of pergolide upon visual motion perception versus cabergoline (and placebo), the observed pergolide effect could have been mediated either by specific D1 activity or a combined D1/D2 action. This ambiguity of pergolide action arises because some dopamine-mediated responses require the synergistic coactivation of D1 and D2 receptors (Hasbi et al., 2011). The observed synergistic D1/D2 activity has a molecular correlate because D1 and D2 receptors may form heteromers (George and O'Dowd, 2007) with unique signaling pathways distinct from D1 or D2 receptors. Pergolide and cabergoline also display serotonergic agonist activity, primarily at 5HT2 receptors (Millan et al., 2002); however, the relevant (albeit scarce) literature does not support serotonin mediation of our 
A

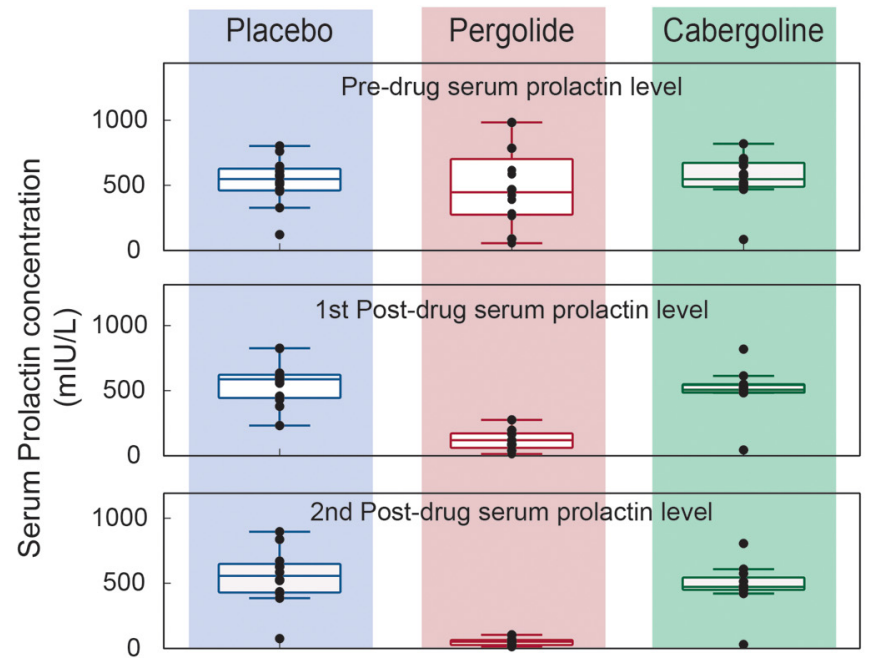

B

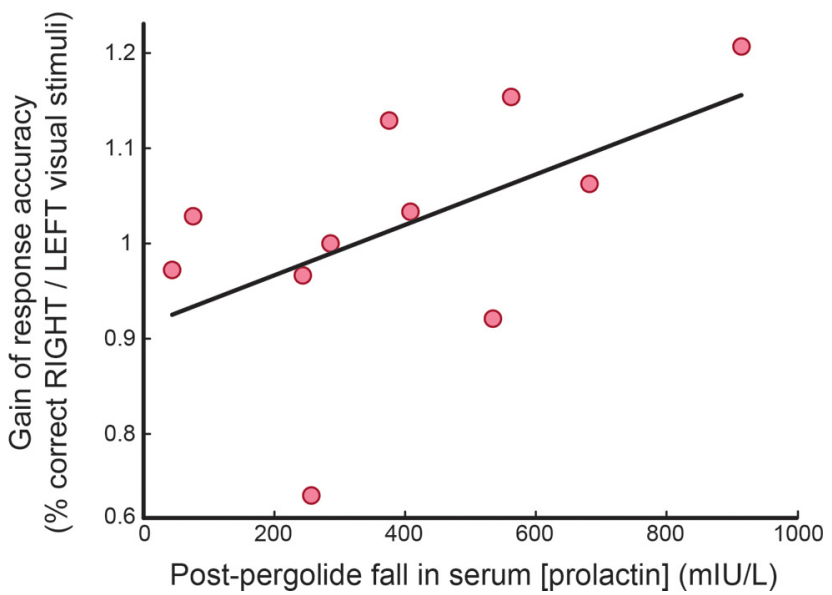

Figure 5. Assessing dopamine agonist bioavailability. $A$, Release of prolactin by the anterior pituitary gland is inhibited by dopamine, so a reduction in serum prolactin levels can act as a surrogate marker for the bioavailability of orally administered dopamine receptor agonists. Note that, to reduce nausea, we preadministered domperidone (over $3 \mathrm{~d}$ ), a selective D2 receptor antagonist that does not penetrate the blood-brain barrier. Because the pituitary gland is perfused by systemic blood, domperidone will tend to counteract dopamine agonists that possess primarily D2 agonist activity (e.g., cabergoline, a selective D2 agonist), but not agonists with activity at other dopamine receptors (e.g., pergolide, a mixed D1/D2 receptor agonist). Correspondingly, for all subjects in Experiment 2, administration of placebo or cabergoline did not affect the level of prolactin across the duration of the experimental session. In contrast, pergolide significantly reduced prolactin serum concentration after its absorption compared with its baseline level. $\boldsymbol{B}$, Furthermore, this change in prolactin correlated with the gain in the subjects' response rate (nonoverlapping/overlapping).

observed pergolide effect (Waterhouse et al., 1990; Horton and Trobe, 1999; Carter et al., 2004).

The use of TMS could affect subjects' performances in a nonspecific manner via effects upon arousal and/or directing attention. A TMS pulse is associated with a loud click and for pulses that precede the presentation of the visual stimulus, there could be an improvement in the subject's performance simply as a result of the alerting effect of the click. Suprathreshold TMS in the left visual cortex also induces a right hemifield phosphene that can direct attention toward or away from the visual stimulus and thus modulate sensory discrimination on this basis alone. Our results, however, indicate that, when the TMS preceded the stimulus, there was no statistical difference between the proportion of correct responses in the overlapping versus nonoverlapping conditions. Furthermore, there was no effect of time in the nonoverlapping condition, which served as our control condition on the response rate, indicating that TMS was not alerting or directing the attention of the subject.

Another potential confound is the effect of dopaminergic activation in visual pathways. For example, the retina contains dopaminergic terminals (Kolb et al., 1992; Zhang et al., 2007), so reduced or increased dopamine may affect retinal function. To control for these effects, in Experiment $B$, we calibrated each participant's baseline RDK performance and TMS parameters at the start of each session, effectively removing any changes in baseline cortical or retinal excitability that could arise from the different drug conditions. Notably, however,
Normalised best chi-square
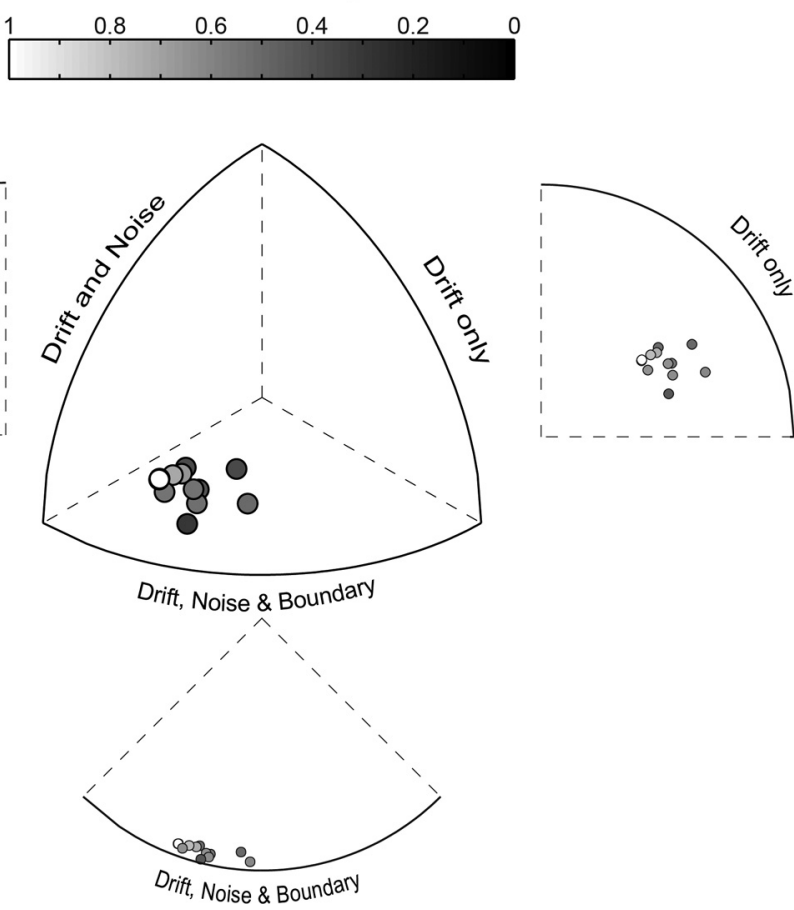

Figure 6. The inclusion of noise variance is essential to account for the effects of dopamine on a drift-diffusion model of perceptual decision making. Shown is a tri-plot with $\chi^{2}$ values corrected by degrees of freedom for the three models: one in which drift rate only is fit; one in which drift and noise variance are fit; and one in which drift rate, noise variance, and boundary position are fit. The three axes vary from 0 to 1 and the points are color coded by the best $\chi^{2}$ value for each subject.

there were no differences across conditions of either the absolute TMS intensity (indicating stable baseline visual cortical excitability) or the percentage coherence in the RDK display needed to obtain the required performance (indicating stable baseline visual perceptual performance). Therefore, the only difference across conditions noted was for the pergolide condition when the TMS and visual stimulus overlapped in time and space. This implies 
that pergolide-related dopaminergic activation confers an advantage in sensory performance only when the sensory SNR is lowered-in the present study, by TMS.

The brain must deal with noise at all stages of sensory processing. Noise is ubiquitous in the environmental signals that our brains attempt to measure, as amply demonstrated by the challenging nature of discerning another person's speech above the hubbub of a cocktail party. Indeed, as a result of noisy cellular processes such as ion channel activity, the sensory transduction process that converts the stimulus to nervous impulses, as well as the subsequent processing in sensory circuits in the CNS, all contribute to noise in the signal and thus affect final outputs such as decision making. Indeed, aging and brain disorders such as migraine, schizophrenia, and PD all show evidence of elevated neuronal noise and impaired sensory processing (Amick et al., 2003; Stebbins et al., 2004; Winterer and Weinberger, 2004; Armstrong, 2011; Almer et al., 2012; Kim et al., 2013; Bates and Wolbers, 2014). For example, compared with healthy controls, schizophrenic patients are less able to maintain visual motion discrimination performance in the face of increased noise in the visual motion signal (Chen et al., 2014). In contrast, we increased noise in cerebral cortical circuits directly by applying visual cortical TMS. The detrimental effect of such noise on visual processing resulted in worsened visual motion direction discrimination when the noise signal (TMS) overlapped the visual stimulus in time and space (in the relevant brain region), but only for the placebo and cabergoline and not the pergolide condition.

One means by which D1/2 receptor (pergolide) activation could attenuate the effect of noise upon visual motion discrimination might be by reducing the responsiveness of the neuronal population such that suprathreshold TMS input is effectively rendered subthreshold. Indeed, subthreshold TMS enhances visual perceptual performance to boost the detection of visual stimuli (Abrahamyan et al., 2011). However, we found no difference in baseline TMS intensities between drug conditions determined during the calibration phase, potentially excluding changes in responsiveness to TMS as an explanation for our results. Critically, however, in the baseline calibration, there was either TMS or visual stimulation but not both. Because brain function is state dependent, the altered TMS responsiveness with pergolide may pertain to the condition of concurrent TMS and visual input (Silvanto and Muggleton, 2008).

That D1 (or combined D1/D2) receptor activation improves sensory perceptual performance in a state-dependent manner, such as during high sensory noise, could provide a rationale for combining dopamine agonists with physical therapy in patients with chronic sensory disorders. A key principle of physical therapy is the use of repetition to improve the performance of a task that is impaired as a result of disease (or prior insult). In the visual domain, such practice is called visual perceptual learning (VPL; Sasaki et al., 2010). Because VPL improves perceptual performance, for example, visual motion discrimination, then combining VPL with D1 or D1/D2 receptor activation could enhance VPL. This could thus benefit patients such as those with stroke affecting the visual cortex, in whom there is an impairment of visual perception in the visual space encoded by the damaged cortex. Fortuitously, VPL can improve visual perception, including visual motion discrimination, after visual cortical damage (Das et al., 2014). Critically, impaired visual perception after visual cortical damage is linked to increased internal noise (Hayes and Merigan, 2007) and, conversely, VPL-related improvement in visual perceptual performance is related to a reduction in internal noise (Cavanaugh et al., 2015). Our data thus offer a rationale in combin- ing dopaminergic agonists to reduce internal noise to boost visual cortical lesion patients' response to VPL (Das et al., 2014).

Our data may also have implications for human diseases with altered brain dopamine processing such as PD and schizophrenia. PD patients are deficient in brain dopamine and commonly display visual perceptual abnormalities, with frank hallucinations affecting up to one-third of PD patients (Sanchez-Ramos et al., 1996). Interestingly, these visual symptoms are more likely in low light when there is high visual stimulus noise. The brain mechanisms underlying visual hallucinations in PD are unknown but are correlated with abnormal prefrontal functioning (Stebbins et al., 2004). In fact, the prefrontal cortex modulates visual cortex functioning because pharmacological antagonism of prefrontal dopamine D1 receptors (but not D2 receptors) enhanced neuronal response in primate area V4 to a similar degree as attention. Together with our data, in high noise states, D1 agonists (via fronto-occipital circuits) should lower visual neuronal responsiveness and thus improve information processing. However, PD patients typically display worsened visual symptoms with dopaminergic administration. It is possible that deficits in frontooccipital pathways in PD may attenuate (or reverse) the beneficial effects of prefrontal dopamine D1 activation upon visual perceptual performance that we observed in healthy subjects. This supposition is supported by findings in schizophrenia, a disease of elevated brain dopamine signaling. Schizophrenic patients, who characteristically show an impairment of visual motion perception, display a reversal of the normal fronto-occipital activation pattern during fMRI when observing visual motion (Chen et al., 2008). Therefore, we suggest that combining dopamine D1 (or D1/D2) activation with neuromodulation of fronto-occipital circuits could treat PD-related hallucinations.

In summary, we have shown that pergolide dopaminergic activation improves visual motion discrimination performance under conditions of increased sensory noise in the visual cortex. This, together with the well documented effects of dopamine upon brain plasticity and learning (Wolf et al., 2003; Hansen and ManahanVaughan, 2014), suggests that combining physical therapy regimens with D1 (or mixed D1/D2) agonists could enhance both the acute saliency of the training visual signal and the long-term effects upon brain plasticity mechanisms (e.g., VPL), in effect "turbo charging" rehabilitation regimens for brain injury.

\section{References}

Abrahamyan A, Clifford CW, Arabzadeh E, Harris JA (2011) Improving visual sensitivity with subthreshold transcranial magnetic stimulation. J Neurosci 31:3290-3294. CrossRef Medline

Agúndez JA, García-Martín E, Alonso-Navarro H, Jiménez-Jiménez FJ (2013) Anti-Parkinson's disease drugs and pharmacogenetic considerations. Expert Opin Drug Metab Toxicol 9:859-874.

Almer Z, Klein KS, Marsh L, Gerstenhaber M, Repka MX (2012) Ocular motor and sensory function in Parkinson's disease. Ophthalmology 119: 178-182. CrossRef Medline

Amassian VE, Cracco RQ, Maccabee PJ, Cracco JB, Rudell A, Eberle L (1989) Suppression of visual perception by magnetic coil stimulation of human occipital cortex. Electroencephalogr Clin Neurophysiol 74:458-462. CrossRef Medline

Amick MM, Cronin-Golomb A, Gilmore GC (2003) Visual processing of rapidly presented stimuli is normalized in Parkinson's disease when proximal stimulus strength is enhanced. Vis Res 43:2827-2835. CrossRef Medline

Arieli A, Sterkin A, Grinvald A, Aertsen A (1996) Dynamics of ongoing activity: explanation of the large variability in evoked cortical responses. Science 273:1868-1871. CrossRef Medline

Armstrong RA (2011) Visual symptoms in Parkinson's disease. Parkinsons Dis 2011:908306. CrossRef Medline

Bates SL, Wolbers T (2014) How cognitive aging affects multisensory inte- 
gration of navigational cues. Neurobiol Aging 35:2761-2769. CrossRef Medline

Beckers G, Hömberg V (1992) Cerebral visual motion blindness: transitory akinetopsia induced by transcranial magnetic stimulation of human area V5. Proc Biol Sci 249:173-178.

Ben-Jonathan N (1985) Dopamine: a prolactin-inhibiting hormone. Review. Endocr Rev 6:564-589.

Borison HL, Borison R (1986) Motion sickness reflex arc bypasses the area postrema in cats. Exp Neurol 92:723-737.

Braddick O (1974) A short-range process in apparent motion. Vis Res 14: 519-527. CrossRef Medline

Brainard DH (1997) The Psychophysics Toolbox. Spat Vis 10:433-436. CrossRef Medline

Carter OL, Pettigrew JD, Burr DC, Alais D, Hasler F, Vollenweider FX (2004) Psilocybin impairs high-level but not low-level motion perception. Neuroreport 15:1947-1951. CrossRef Medline

Cavanaugh JR, Bair W, Movshon JA (2002) Nature and interaction of signals from the receptive field center and surround in macaque V1 neurons. J Neurophysiol 88:2530-2546. CrossRef Medline

Cavanaugh MR, Zhang R, Melnick MD, Das A, Roberts M, Tadin D, Carrasco M, Huxlin KR (2015) Visual recovery in cortical blindness is limited by high internal noise. J Vis 15:9. CrossRef Medline

Chen Y, Grossman ED, Bidwell LC, Yurgelun-Todd D, Gruber SA, Levy DL, Nakayama K, Holzman PS (2008) Differential activation patterns of occipital and prefrontal cortices during motion processing: evidence from normal and schizophrenic brains. Cogn Affect Behav Neurosci 8:293303. CrossRef Medline

Chen Y, Norton D, McBain R (2014) Effects of domain-specific noise on visual motion processing in schizophrenia. PLoS One 9:e99031. CrossRef Medline

Cowey A, Walsh V (2001) Tickling the brain: studying visual sensation, perception and cognition by transcranial magnetic stimulation. Prog Brain Res 134:411-425. CrossRef Medline

Das A, Tadin D, Huxlin KR (2014) Beyond blindsight: properties of visual relearning in cortically blind fields. J Neurosci 34:11652-11664. CrossRef Medline

Del Dotto P, Bonuccelli U (2003) Clinical pharmacokinetics of cabergoline. Review. Clin Pharmacokinet 42:633-645.

El-Shamayleh Y, Movshon JA (2011) Neuronal responses to texturedefined form in macaque visual area V2. J Neurosci 31:8543-8555. CrossRef Medline

Ermentrout GB, Galán RF, Urban NN (2008) Reliability, synchrony and noise. Trends Neurosci 31:428-434. CrossRef Medline

Fariello RG (1998) Pharmacodynamic and pharmacokinetic features of Cabergoline. Rationale for use in Parkinson's disease. Review. Drugs 55(Suppl 1):10-16.

Frucht S, Rogers JD, Greene PE, Gordon MF, Fahn S (1999) Falling asleep at the wheel: motor vehicle mishaps in persons taking pramipexole and ropinirole. Neurology 52:1908-1910.

George SR, O'Dowd BF (2007) A novel dopamine receptor signaling unit in brain: heterooligomers of D1 and D2 dopamine receptors. ScientificWorldJournal 7:58-63. Medline

Hansen N, Manahan-Vaughan D (2014) Dopamine D1/D5 receptors mediate informational saliency that promotes persistent hippocampal longterm plasticity. Cereb Cortex 24:845-858. CrossRef Medline

Hasbi A, O'Dowd BF, George SR (2011) Dopamine D1-D2 receptor heteromer signaling pathway in the brain: emerging physiological relevance. Mol Brain 4:26. CrossRef Medline

Hayes RD, Merigan WH (2007) Mechanisms of sensitivity loss due to visual cortex lesions in humans and macaques. Cereb Cortex 17:1117-1128. Medline

Herbert M, Johns MW, Doré C (1976) Factor analysis of analogue scales measuring subjective feelings before and after sleep. Br J Med Psychol 49:373-379.

Horton JC, Trobe JD (1999) Akinetopsia from nefazodone toxicity. Am J Ophthalmol 128:530-531. CrossRef Medline

Jansen PA, Herings RM, Samson MM, De Vreede PL, Schuurmans-Daemen LM, Hovestadt A, Verhaar HJ, Van Laar T (2001) Quick titration of pergolide in cotreatment with domperidone is safe and effective. Clin Neuropharmacol 24:177-180. CrossRef Medline

Kim J, Norton D, McBain R, Ongur D, Chen Y (2013) Deficient biological motion perception in schizophrenia: results from a motion noise paradigm. Front Psychol 4:391. CrossRef Medline

Kolb H, Linberg KA, Fisher SK (1992) Neurons of the human retina: a Golgi study. J Comp Neurol 318:147-187. CrossRef Medline
Kroener S, Chandler LJ, Phillips PE, Seamans JK (2009) Dopamine modulates persistent synaptic activity and enhances the signal-to-noise ratio in the prefrontal cortex. PLoS One 4:e6507. CrossRef Medline

Laduron PM, Leysen JE (1979) Domperidone, a specific in vitro dopamine antagonist, devoid of in vivo central dopaminergic activity. Biochem Pharmacol 28:2161-2165.

Millan MJ, Maiofiss L, Cussac D, Audinot V, Boutin JA, Newman-Tancredi A (2002) Differential actions of antiparkinson agents at multiple classes of monoaminergic receptor. I. A multivariate analysis of the binding profiles of 14 drugs at 21 native and cloned human receptor subtypes. J Pharmacol Exp Ther 303:791-804. CrossRef Medline

Miller AD, Leslie RA (1994) The area postrema and vomiting. Review. Front Neuroendocrinol 15:301-320.

Müller U, von Cramon DY, Pollmann S (1998) D1- versus D2-receptor modulation of visuospatial working memory in humans. J Neurosci 18: 2720-2728. Medline

Norbury A, Manohar S, Rogers RD, Husain M (2013) Dopamine modulates risk-taking as a function of baseline sensation-seeking trait. J Neurosci 33:12982-12986. CrossRef Medline

Noudoost B, Moore T (2011) Control of visual cortical signals by prefrontal dopamine. Nature 474:372-375. CrossRef Medline

PelliDG (1997) TheVideoToolbox software for visual psychophysics: transforming numbers into movies. Spat Vis 10:437-442. CrossRef Medline

Rascol O (1999) Dopamine agonists: what is the place of the newer compounds in the treatment of Parkinson's disease? Review. J Neural Transm Suppl 55:33-45.

Ratcliff R, McKoon G (1988) A retrieval theory of priming in memory. Psychol Rev 95:385-408. CrossRef Medline

Reichmann H, Herting B, Miller A, Sommer U (2003) Switching and combining dopamine agonists. J Neural Transm 110:1393-1400. CrossRef Medline

Ruzzoli M, Marzi CA, Miniussi C (2010) The neural mechanisms of the effects of transcranial magnetic stimulation on perception. J Neurophysiol 103:2982-2989. CrossRef Medline

Sanchez-Ramos JR, Ortoll R, Paulson GW (1996) Visual hallucinations associated with Parkinson disease. Arch Neurol 53:1265-1268. CrossRef Medline

Sasaki Y, Nanez JE, Watanabe T (2010) Advances in visual perceptual learning and plasticity. Nat Rev Neurosci 11:53-60. CrossRef Medline

Seemungal BM, Guzman-Lopez J, Arshad Q, Schultz SR, Walsh V, Yousif N (2013) Vestibular activation differentially modulates human early visual cortex and V5/MT excitability and response entropy. Cereb Cortex 23:12-19.

Silvanto J, Muggleton NG (2008) Testing the validity of the TMS statedependency approach: targeting functionally distinct motion-selective neural populations in visual areas V1/V2 and V5/MT+. Neuroimage 40: 1841-1848. CrossRef Medline

Stebbins GT, Goetz CG, Carrillo MC, Bangen KJ, Turner DA, Glover GH, Gabrieli JD (2004) Altered cortical visual processing in PD with hallucinations: an fMRI study. Neurology 63:1409-1416. CrossRef Medline

Stefanini E, Clément-Cormier Y, Vernaleone F, Devoto P, Marchisio AM, Collu R (1981) Sodium-dependent interaction of benzamides with dopamine receptors in rat and dog anterior pituitary glands. Neuroendocrinology 32:103-107.

Stewart L, Ellison A, Walsh V, Cowey A (2001) The role of transcranial magnetic stimulation (TMS) in studies of vision, attention and cognition. Acta Psychol (Amst) 107:275-291.

Waterhouse BD, Azizi SA, Burne RA, Woodward DJ (1990) Modulation of rat cortical area 17 neuronal responses to moving visual stimuli during norepinephrine and serotonin microiontophoresis. Brain Res 514:276292. CrossRef Medline

Watson AB, Pelli DG (1983) QUEST: a Bayesian adaptive psychometric method. Percept Psychophys 33:113-120.

Winterer G, Weinberger DR (2004) Genes, dopamine and cortical signal-to-noise ratio in schizophrenia. Trends Neurosci 27:683-690. CrossRef Medline

Wolf ME, Mangiavacchi S, Sun X (2003) Mechanisms by which dopamine receptors may influence synaptic plasticity. Ann N Y Acad Sci 1003:241249. CrossRef Medline

Zaldivar D, Rauch A, Whittingstall K, Logothetis NK, Goense J (2014) Dopamine-induced dissociation of BOLD and neural activity in macaque visual cortex. Curr Biol 24:2805-2811. CrossRef Medline

Zhang DQ, Zhou TR, McMahon DG (2007) Functional heterogeneity of retinal dopaminergic neurons underlying their multiple roles in vision. J Neurosci 27:692-699. CrossRef Medline 